\title{
EVALUACIÓN DE TEXTURA DEL SUELO CON ESPECTROSCOPÍA DE INFRARROJO CERCANO EN UN OXISOL DE COLOMBIA
}

\author{
Near-infrared spectroscopic assessment of soil texture in an oxisol \\ of the eastern plains of Colombia
}

\author{
Jesús H. Camacho-Tamayo', Nathalia M. Forero-Cabrera², \\ Leonardo Ramírez-López ${ }^{3} \&$ Yolanda Rubiano ${ }^{4}$
}

Camacho-Tamayo, J., Forero-Cabrera, N., Ramírez-López, L. \& Rubiano, Y. (2017). Evaluación de textura del suelo con espectroscopía de infrarrojo cercano en un oxisol de Colombia. Colombia Forestal, 20(1),5-18

Recepción: 17 de noviembre de 2015

\section{Resumen}

Técnicas como la espectroscopía de infrarrojo cercano (NIR) se pueden utilizar para identificarlas clases y propiedades de los suelos con buena precisión. El objetivo de este estudio fue calibrar modelos para predecir el contenido de arcilla, limo y arena de un Typic Hapludox por espectroscopia NIR. El estudio se realizó en la Estación Experimental de Carimagua situado en el municipio de Puerto Gaitán, Meta, Colombia. Se utilizó un diseño de red rígida, se tomaron 1200 muestras en una superficie aproximada de 5100 ha. La elaboración de los modelos se hizo mediante regresión por mínimos cuadrados parciales. Se obtuvieron modelos con baja representatividad para contenidos de arena y limo, con valores de $\mathrm{R}^{2}$ (0.41 y 0.34 , respectivamente). El modelo para el contenido de arcilla mostró un alto $\mathrm{R}^{2}(0.76)$. Para la arcilla fue posible la elaboración de mapas digitales y espectro-digitales similares. Los resultados encontrados para el contenido de arcilla indican que los análisis de laboratorio se pueden sustituir, en gran parte, por los modelos espectrales. En el caso de la
Aceptación: 24 de octubre de 2016

arena y el limo, sería conveniente mejorar el modelo para que, en el futuro, los análisis de laboratorio puedan ser sustituidos para esta clase de suelo.

Palabras clave: mapeo de suelos, reflectancia difusa, Typic Hapludox.

\begin{abstract}
Techniques such as near infrared spectroscopy (NIR) can be used to identify classes and properties of soils with good precision. The aim of this study was to calibrate models to predict the content of clay, silt and sand of a Typic Hapludox by NIR spectroscopy. The study was carried out in the Carimagua Experimental Station located in the municipality of Puerto Gaitán, Meta, Colombia. A grid design was used, 1200 samples were collected in an area of 5100 ha. The development of the models have made by partial least squares regression. A low representatively models were obtained for sand and silt contents, with values of $R^{2}$ ( 0.41 and 0.34 , respectively).The clay model content showed a high $\mathrm{R}^{2}(0.76)$. For the clay was possible the development of similar digital maps and
\end{abstract}

1 Universidad Nacional de Colombia. Bogotá, Colombia. jhcamachot@unal.edu.co.

2 Universidad Nacional de Colombia. Bogotá, Colombia.nmforeroc@unal.edu.co. Autor para correspondencia.

3 Institute of Terrestrial Ecosystems, Swiss Federal Institute of Technology (ETH); Swiss Federal Institute for Forest, Snow and Landscape Research. Zurich and Birmensdorf, Switzerland. leonardo.ramirez@usys.ethz.ch

4 Universidad Nacional de Colombia. Bogotá, Colombia.yrubianos@unal.edu.co 
digital spectrum maps. The results for the clay content indicate that laboratory analysis can be substituted, in large part by spectral models. In the case of sand and silt, in the future, it would be convenient to improve the model for substituting the laboratory analysis for this class of soil.

Key words: soil mapping, diffuse reflectance, Typic Hapludox.

\section{INTRODUCCIÓN}

La textura del suelo es una propiedad de gran interés que se relaciona directamente con los procesos de degradación y potencial de producción (White, 2005). La caracterización de un suelo es esencial para determinar su apropiado manejo y realizar planificación agrícola. La textura indica el contenido de partículas de arena, limo y arcilla en el suelo. Así mismo, influye en otras propiedades como la densidad aparente, la porosidad y, por lo tanto, el movimiento y el almacenamiento de fluidos (agua y aire) en el suelo (Lacasta et al., 2005).

La región que comprende los Llanos Orientales de Colombia presenta un crecimiento cercano al $54 \%$ del área sembrada durante el periodo 2004-2014 (MinAgricultura, 2016). El estudio de los suelos de la región es importante y así mismo la implementación de nuevas técnicas de muestreo, laboratorio y análisis se hace necesaria. Los suelos de la zona de Carimagua estructuralmente van desde arcillosos hasta arenosos, pasando por los francos. Todos ellos se caracterizan por importantes contenidos de hierro, lo cual se advierte por el color rojo de su perfil, pues se trata de oxisoles. Otra característica de estos suelos son los altos niveles de aluminio, elemento que en altos contenidos puede ser tóxico para las plantas e inhibir el crecimiento de raíces (García, 2009).

Actualmente, la técnica de espectroscopía de reflectancia se ha propuesto como una alternativa para reemplazar los métodos convencionales en la determinación de las propiedades del suelo. Esta técnica presenta un gran potencial por ser de menor costo y mucho más rápida que las técnicas convencionales. Los métodos analíticos utilizados demanda largas horas de recolección de datos para obtener los resultados y requiere insumos químicos que pueden generar impactos ambientales (Nanni \& Demattê, 2006; Sousa Junior et al., 2011) observaron que la determinación de la distribución del tamaño de partícula de una muestra de suelo seco y tamizado por el método de Bouyoucos puede demorar hasta 48 horas, mientras que la misma determinación hecha con información espectral del suelo VIS-NIR -SWIR (visible e infrarrojo cercano de onda corta infrarroja, 400-2500 nm) toma algunos minutos.

La espectroscopía de reflectancia estudia la energía que refleja un material en función de la longitud de onda, como parte del fraccionamiento de la energía incidente debido a los procesos electrónicos y vibratorios relacionados con minerales que contienen hierro (por ejemplo, hematita y goethita). Si bien las bandas fundamentales de vibración se encuentran principalmente en regiones del infrarrojo medio y lejano, los procesos vibratorios producen características en la región del infrarrojo cercano (NIR, intervalo entre $780 \mathrm{~nm}$ y 2500 nm) debido a la excitación de armónicos y la combinación de los tonos de los periodos fundamentales de grupos aniónicos (Ramírez et al., 2005). En resumen, la energía reflejada por un cuerpo sólido es una combinación de dos clases de reflexión (especular o difusa) y su magnitud depende tanto del tamaño de partículas, estructura, mineralogía, contenido de agua del suelo, micro relieve, como otras características (Camacho-Tamayo et al., 2014).

Para el caso específico de la determinación de textura del suelo, la influencia en el VIS-NIR se produce como la intensidad de la reflectancia sobre la firma espectral (albedo), pues la mayoría de los suelos con contenido de arcilla presentan 
una menor reflexión de la energía electromagnética incidente (Bellinaso et al., 2010). Además, los minerales de arcilla tales como filosilicatos y óxidos, hidróxidos y oxi-hidróxidos de hierro y aluminio presentes en el suelo son los responsables de las características de absorción del espectro de reflectancia por VIS-NIR (Stenberg et al., 2010). La respuesta espectral de cuarzo, mineral abundante en la fracción arena en el VIS-NIR, se caracteriza por un albedo mayor y ausencia de características de absorción, que se observa por lo general en el espectro de los suelos arenosos (Bellinaso et al., 2010). Con base en lo expuesto, el objetivo del presente estudio fue calibrar y validar modelos para predecir el contenido de arcilla, limo y arena de un Typic Hapludox por medio de espectroscopia NIR, como una alternativa a los estudios de textura tradicionales.

\section{MATERIALES Y MÉTODOS}

\section{Área de estudio}

El estudio se realizó en la Estación Experimental de Carimagua, situada en el municipio de Puerto Gaitán, Meta, Colombia, con coordenadas geográficas de $4^{\circ} 37^{\prime} \mathrm{N}$ y $71^{\circ} 19^{\prime} \mathrm{W}$ (figura 1) y una altitud media de $175 \mathrm{~m}$. El uso dominante del lugar es ganadería extensiva con sabana nativa. La zona presenta un relieve ligeramente ondulado con pendientes entre $2 \%$ y $5 \%$, con clima tropical subhúmedo Awf (clasificación Köppen). La temperatura media es de $27.8^{\circ} \mathrm{C}$ y la precipitación media anual de $2240 \mathrm{~mm}$, concentrada entre los meses de abril y diciembre. Predominan los suelos oxisoles altamente agregados, caracterizados por ser fuertemente ácidos $(\mathrm{pH}<5)$.

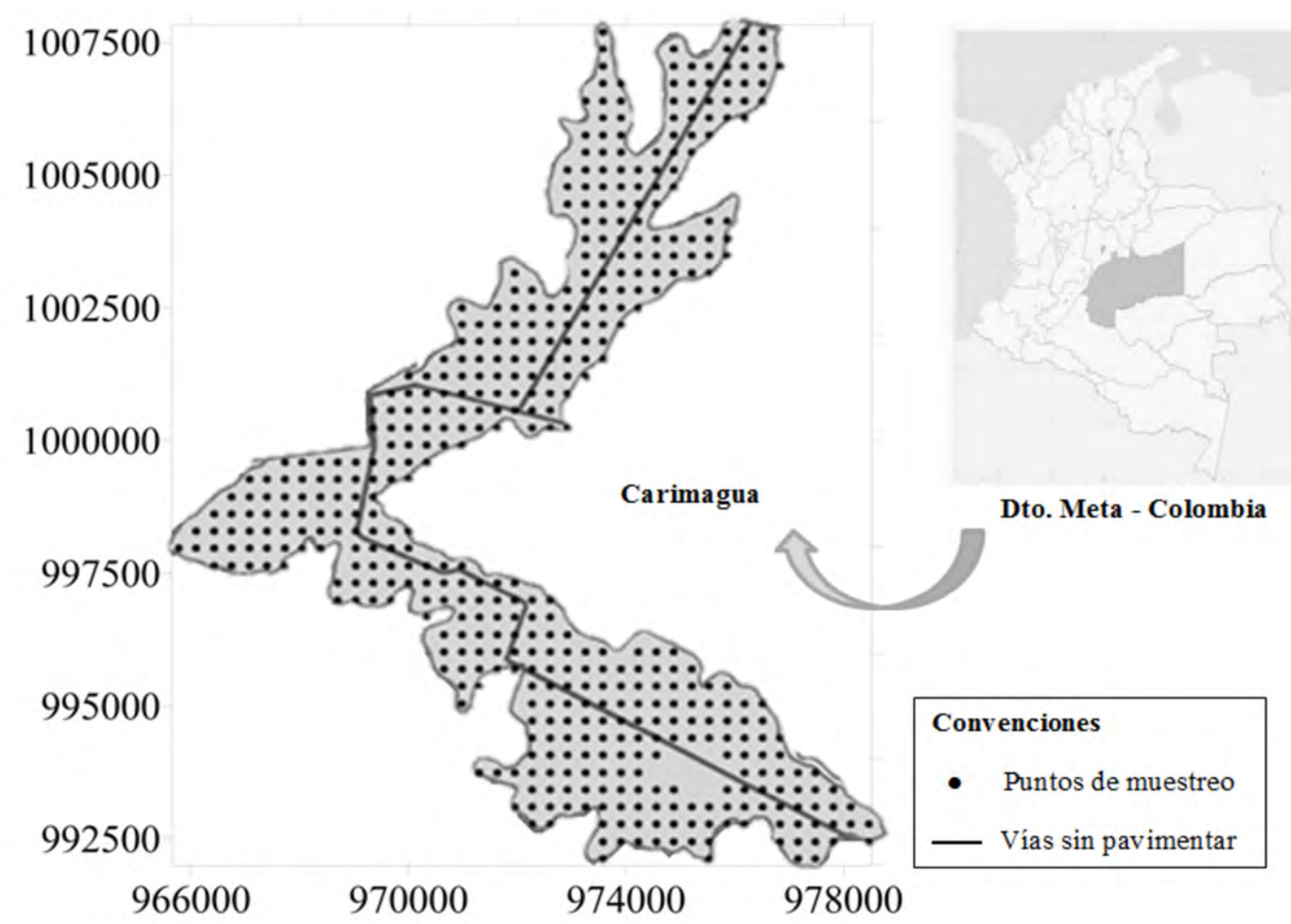

Figura 1. Localización del área de estudio (Coordenadas planas, Sistema de referencia: MAGNA_Colombia_Este). 


\section{Muestreo y análisis de laboratorio}

Se estableció un sistema de malla rígida con puntos ubicados perpendicularmente cada 320 m, donde se elaboraron 470 cajuelas para la toma de muestras en un área de 5100 ha aproximadamente. Con el fin de garantizar observaciones cada 2.5 ha se tomaron adicionalmente 150 muestras con barrenos en un área piloto (correspondiente al $10 \%$ del área de estudio). En cada punto se realizó el muestreo para los horizontes superficial (A) y subsuperficial (B), para un total de 1240 muestras. Todos los puntos fueron georreferenciados con GPS (exactitud de $\pm 1 \mathrm{~m}$ ).

Posteriormente, las muestras fueron secadas a $35^{\circ} \mathrm{C}$ y tamizadas en malla de $2 \mathrm{~mm}$. A partir de esta condición se realizó la adquisición de las curvas espectrales NIR y la determinación de los contenidos de arena, limo y arcilla por el método de Bouyoucos. Las respuestas espectrales se obtuvieron a partir del sensor NIRS 6500 (FOSS NIR Systems, Inc.), que arroja lecturas de la respuesta espectral cada $2 \mathrm{~nm}$, a partir de una media de 64 lecturas (scans) por longitud de onda.

\section{Análisis de datos}

Para la calibración de los modelos se formaron 20 grupos de 50, 100, 150, 200, 250, 300, 350, 400, 450, 500, 550, 600, 650, 700, 750, 800, 850, 900, 950 y 1000 muestras para el grupo número 20, dejando 200 muestras para validar el modelo. La formación de cada grupo se realizó mediante el uso del método de selección de muestras del Hipercubo Latino (cLHS). Este es un procedimiento de muestreo aleatorio estratificado que consiste en seleccionar valores iniciales para la construcción del modelo y estratificar el rango de cada uno de los datos de entrada de este, con el fin de garantizar de esta manera que se seleccionan los valores iniciales de cada rango de los datos de entrada. En esta técnica se necesitan menos muestras para obtener una distribución más representativa de las características de la zona estudiada (Carvalho Junior et al., 2014).
Las respuestas espectrales fueron previamente procesadas, con el objetivo de corregir posibles ruidos o interferencias. Esto se logró a través de técnicas como Detrend, para corregir la tendencia de los datos, SNV (Standard Normal Variation), tratamiento que corrige alteraciones ópticas, y MSC (Multiplicative Signal Correction), para corregir la dispersión múltiple principalmente cuando se definen diversos grupos de muestras. También se utilizaron filtros como Median Filter, Wavelet y Savitzky-Golay, los cuales buscan eliminar parte del ruido a través del suavizado de los puntos

En la calibración de los modelos se utilizó el método "leave one out", que provee información sobre la incertidumbre generada con diferentes factores PLS (mínimos cuadrados parciales), basado en el remuestreo, realizado en la validación cruzada. Se definió el número de factores PLS con base en los resultados de la validación de los modelos, considerando parámetros como el coeficiente de determinación $\left(\mathrm{R}^{2}\right)$, la desviación residual de la predicción (RPD), la raíz del error cuadrático medio(RMSE), el error medio (ME) y la desviación estándar del error (SDE). Este análisis se realizó con el programa de uso libre ParLeS v. 1.0, desarrollado por Viscarra Rossel (2008).

Las correlaciones entre las propiedades del suelo y las longitudes de onda se determinaron empleando la prueba de correlación de Pearson ( $r$ ) y se clasificaron de acuerdo a la propuesta realizada por Bisquerra (2004). Este autor indica que una correlación cercana a uno (1.0) es una correlación perfecta; un valor entre 0.8 y 1.0 corresponde a correlación muy alta; entre 0.6 y 0.8 corresponde a una correlación alta; entre 0.4 y 0.6 sería una correlación moderada; entre 0.2 y 0.4 representa una correlación baja; entre 0 y 0.2 se trata de una correlación muy baja y un valor de cero (0.0) se define como una correlación nula.

Se consideró que el $\mathrm{R}^{2}$ y RMSE deben presentar por lo menos un valor del $95 \%$ de la meseta y el RPD debe estar alrededor de 1.5 o 2.0, con el fin de determinar el número mínimo de muestras necesario para la calibración de los modelos. Los 
parámetros de la calibración de los modelos $\left(\mathrm{R}^{2}\right.$, RMSE y RPD) obtenidos por cada grupo de muestras se ajustaron a funciones de tipo exponencial, sigmoide y sigmoidal Chapman.

\section{Análisis geoestadístico}

Para el caso específico del contenido de arcilla, se calcularon los semivariogramas experimentales, tanto para los datos medidos como para los estimados a partir de los modelos NIR, con los grupos de muestras establecidos. Webster \& Oliver (2007) presentan las características y condiciones que deben cumplir varios modelos teóricos de semivarianza que pueden ajustarse al semivariograma experimental. Estos modelos se pueden dividir en no acotados (lineal, logarítmico y potencial) y acotados (esférico, exponencial y gaussiano), con tres parámetros comunes, el efecto pepita $\left(\mathrm{C}_{0}\right)$, la meseta $\left(\mathrm{C}_{0}+\mathrm{C}_{1}\right)$ y el rango o alcance $(\mathrm{a})$.

El grado de dependencia espacial (GDE) se verificó con la relación entre el efecto pepita y la meseta. Según Cambardella et al. (1994), el GDE se clasifica como fuerte si es mayor que $75 \%$, moderado si esta entre $25 \%$ y $75 \%$ y débil por debajo de $25 \%$. Para que el modelo de correlación espacial describa de manera adecuada la realidad es importante que el efecto pepita no supere el $50 \%$ del valor de la meseta. En casos diferentes, el ruido en las mediciones se explicaría en mayor proporción debido a la variabilidad espacial que a la correlación del fenómeno. Para estos casos el modelo ajustado al semivariograma experimental se denomina como efecto pepita puro (Goovaerts, 1998).
Se seleccionaron modelos teóricos de semivariogramas con base en los indicadores más apropiados según Johann et al. (2010), que son el coeficiente de determinación $\left(R^{2}\right)$ de la ecuación de ajuste, el menor valor de la suma de los cuadrados residuales y en las semejanzas observadas en la validación cruzada entre el valor real y el valor estimado. Para el análisis geoestadístico se empleó el software GS+ v.9 (Gamma Design Software, LLC, Plainwell, MI).

Se realizó la predicción con el método de kriging ordinario a partir de los modelos de los semivariogramas de las propiedades que expresaron dependencia espacial: De acuerdo con Diggle et al. (2000), este método se considera como el mejor estimador lineal insesgado de mínima varianza, útil en la predicción de sitios no muestreados. Los resultados se presentan en mapas digitales, a partir de los datos medidos y mapas espectro-digitales, con base en los datos obtenidos de los modelos calibrados y validados. Teniendo en cuenta que algunas propiedades no mostraban estacionariedad, pues no habían cambios respecto a la media dentro de la zona de estudio, para kriging ordinario se especificó un vecindario de búsqueda local, esto con ayuda del programa Surfer v.9 (Golden Software Inc., Golden, CO).

\section{RESULTADOS}

En la zona de estudio se verifica una textura más fina para el segundo horizonte, el horizonte A presentó una textura franco arcillosa y el horizonte B una textura franco arcillo limosa (Tabla 1).

Tabla 1. Estadística descriptiva para textura en el horizonte A y en el horizonte B de los contenidos de arena, limo y arcilla.

\begin{tabular}{lclllll}
\hline \multicolumn{1}{c}{ Propiedad } & \multicolumn{3}{c}{ Horizonte A } & \multicolumn{3}{c}{ Horizonte B } \\
\hline & Media & Mediana & CV (\%) & Media & Mediana & CV (\%) \\
\hline Arena (\%) & 25.86 & 24.96 & 19.93 & 22.46 & 22.48 & 17.97 \\
Limo (\%) & 45.68 & 46.00 & 10.71 & 42.00 & 42.00 & 12.07 \\
Arcilla (\%) & 28.38 & 28.50 & 16.88 & 35.25 & 35.04 & 13.17 \\
\hline
\end{tabular}

CV: Coeficiente de variación. 
Para los parámetros de calibración de los modelos de los contenidos de arena, limo y arcilla se obtuvo los siguientes resultados.

Tabla 2. Resultados de parámetros obtenidos para los modelos de arena, limo y arcilla.

\begin{tabular}{lccc}
\hline \multicolumn{1}{c}{ Propiedad } & $\mathbf{R}^{\mathbf{2}}$ & RMSE & RPD \\
\hline Arena & 0.34 & 4.75 & 1.22 \\
Limo & 0.41 & 4.04 & 1.30 \\
Arcilla & 0.76 & 2.86 & 2.05 \\
\hline
\end{tabular}

$\mathrm{R}^{2}$ : coeficiente de determinación, RMSE: raíz del error cuadrático medio, RPD: desviación residual de la predicción.
A continuación, se presentan los resultados de las validaciones cruzadas para los grupos de calibración y validación de los contenidos de limo, arena y arcilla.

Para el contenido de arcilla la ecuación de tipo sigmoide fue la que representó mejor el comportamiento de los parámetros $\mathrm{R}^{2}$ y RPD, en el caso del RMSE la ecuación de mejor ajuste fue de tipo sigmoidal de Chapman, dicho esto, se presentan las gráficas para cada uno de los parámetros en función del número de muestras.

La importancia de la variable para la proyección (VIP) se consideró para identificar las regiones
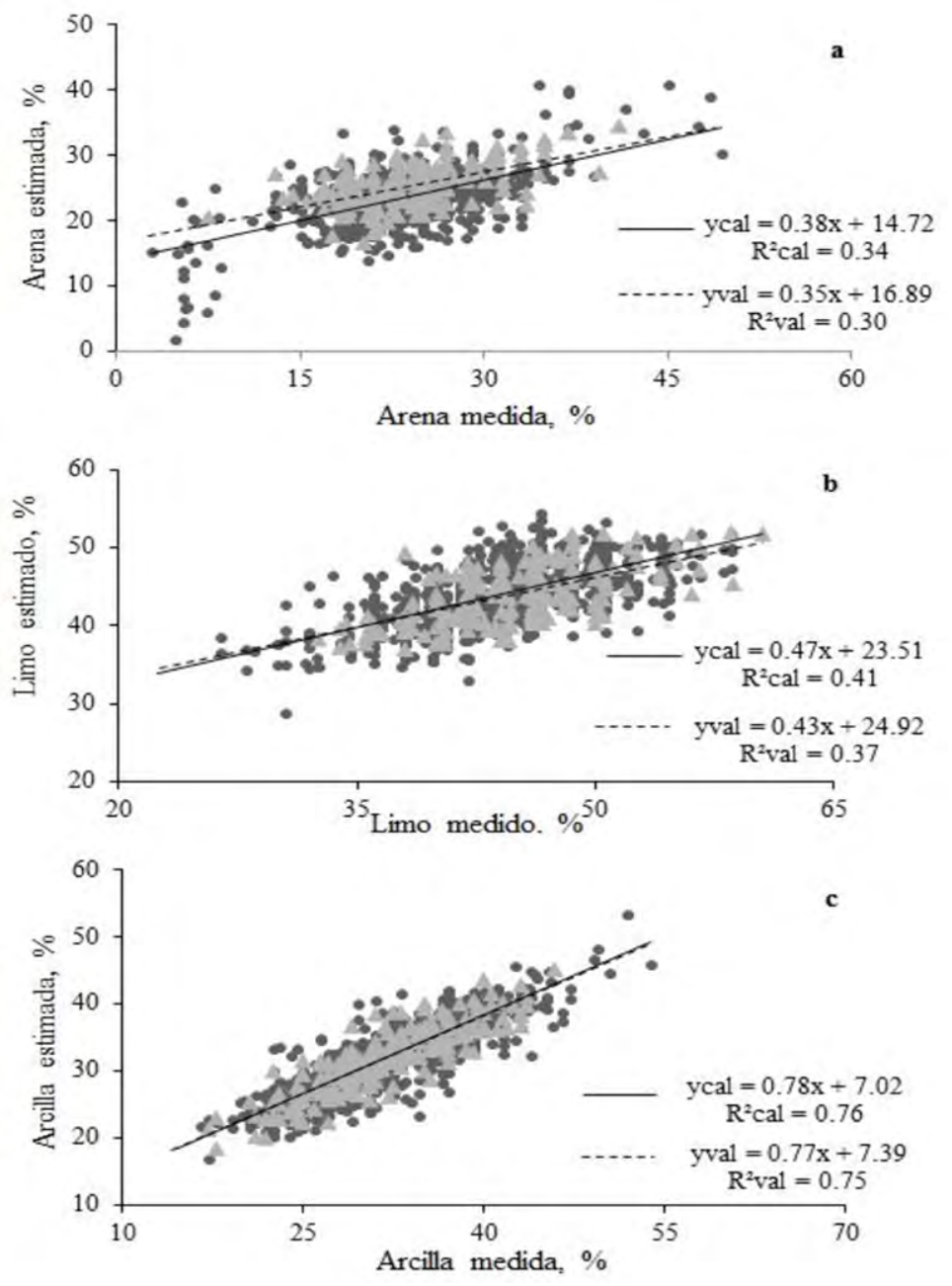

Figura 2. Validación cruzada. a) Resultados para arena, b) Resultados para limo y c) Resultados para arcilla. $\triangle$ Datos de validación; • Datos de calibración. 

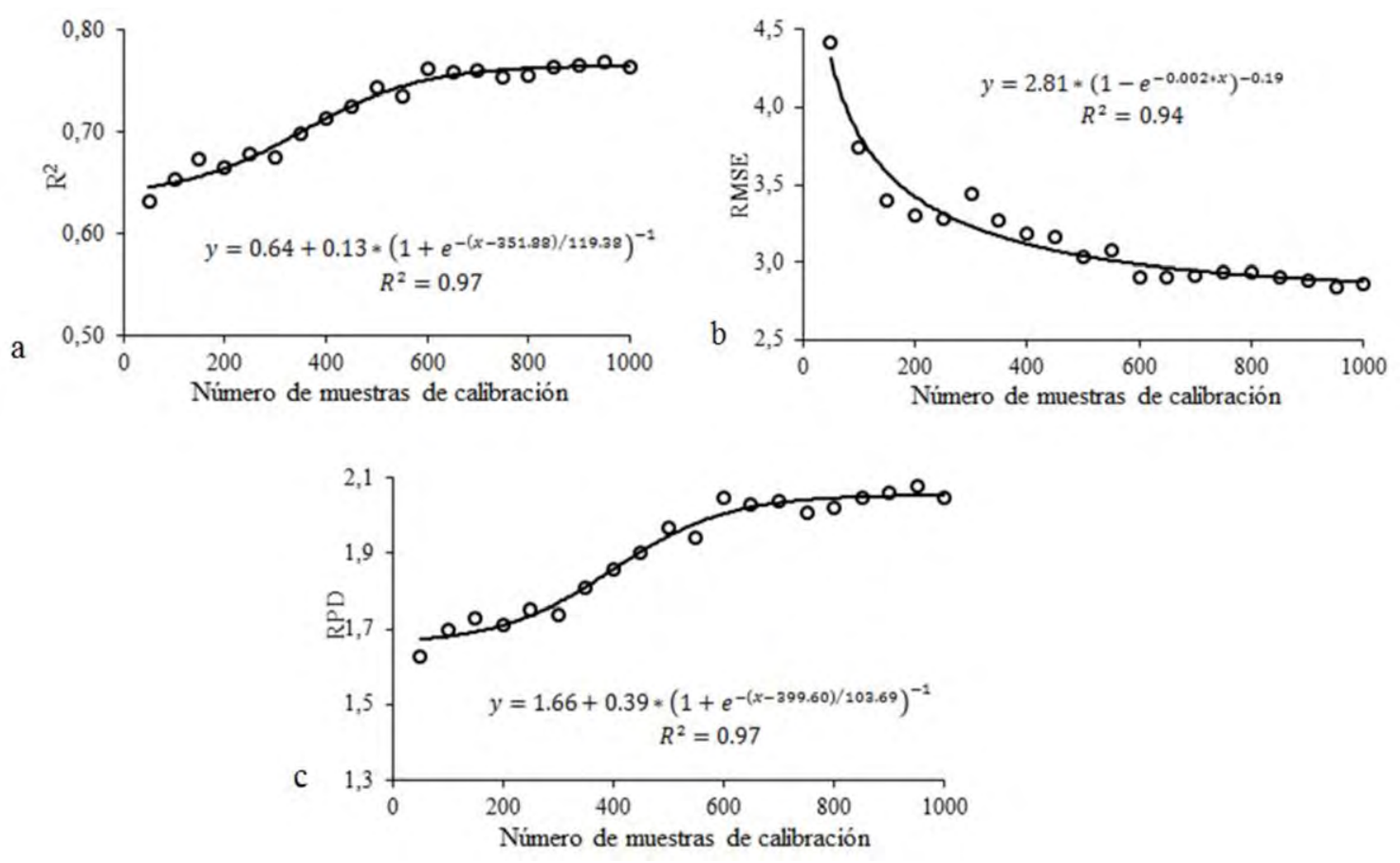

Figura 3. Variación de los parámetros estadísticos en función del número de muestras de calibración para el contenido de arcilla. A variación $\mathrm{R}^{2}$, B variación RMSE y C variación RPD.

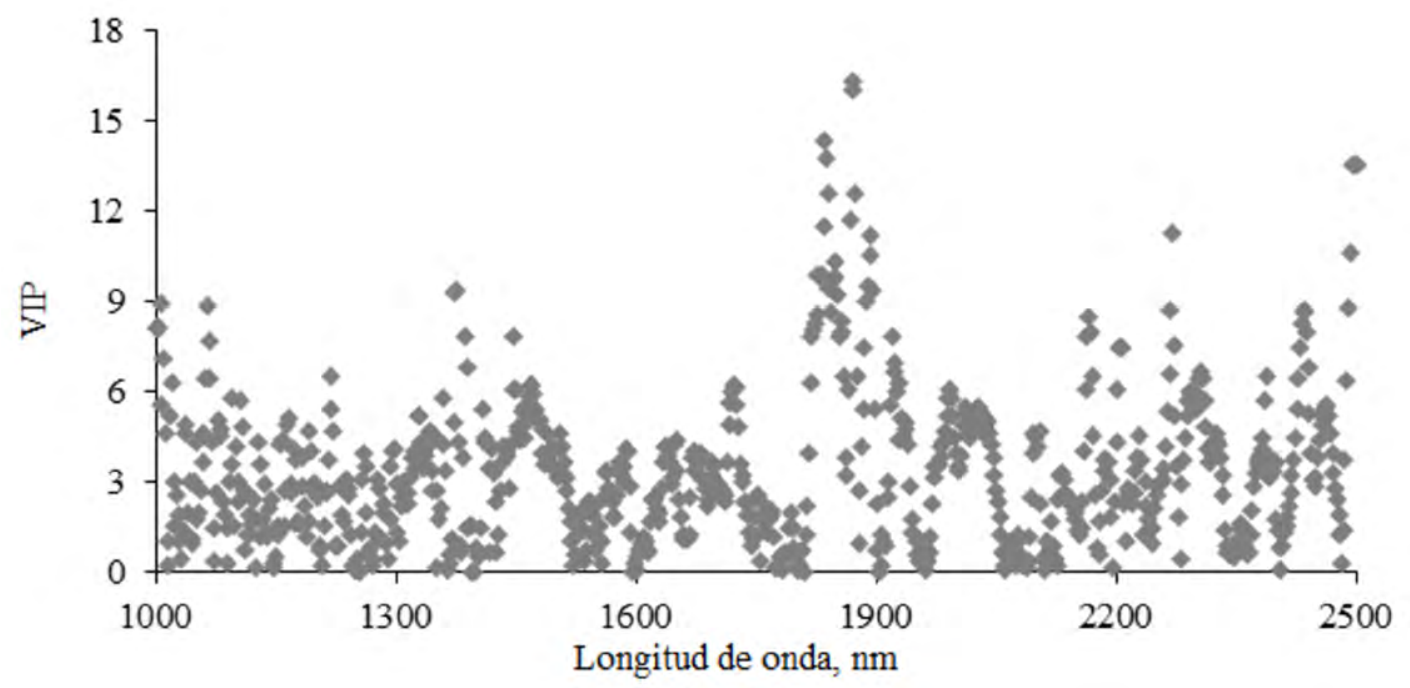

Figura 4. Importancia de la variable para la proyección (VIP) en la respuesta espectral del contenido de arcilla.

de la curva espectral o las longitudes de onda de mayor representatividad en el modelo de contenido de arcilla Wold et al. (2001). La siguiente grafica corresponde al VIP para el contenido de arcilla.
Para el análisis espacial, los resultados obtenidos por método convencional y los estimados a partir del modelo NIR (para 200, 500 y 1000 muestras) se consideraron para la elaboración de 
mapas digitales. Para los dos horizontes se obtuvo que el modelo esférico fue el predominante.

A continuación, se presentan los mapas digitales, mapas espectro digitales y semivariogramas obtenidos para el contenido de arcilla en los dos horizontes de la zona de estudio.
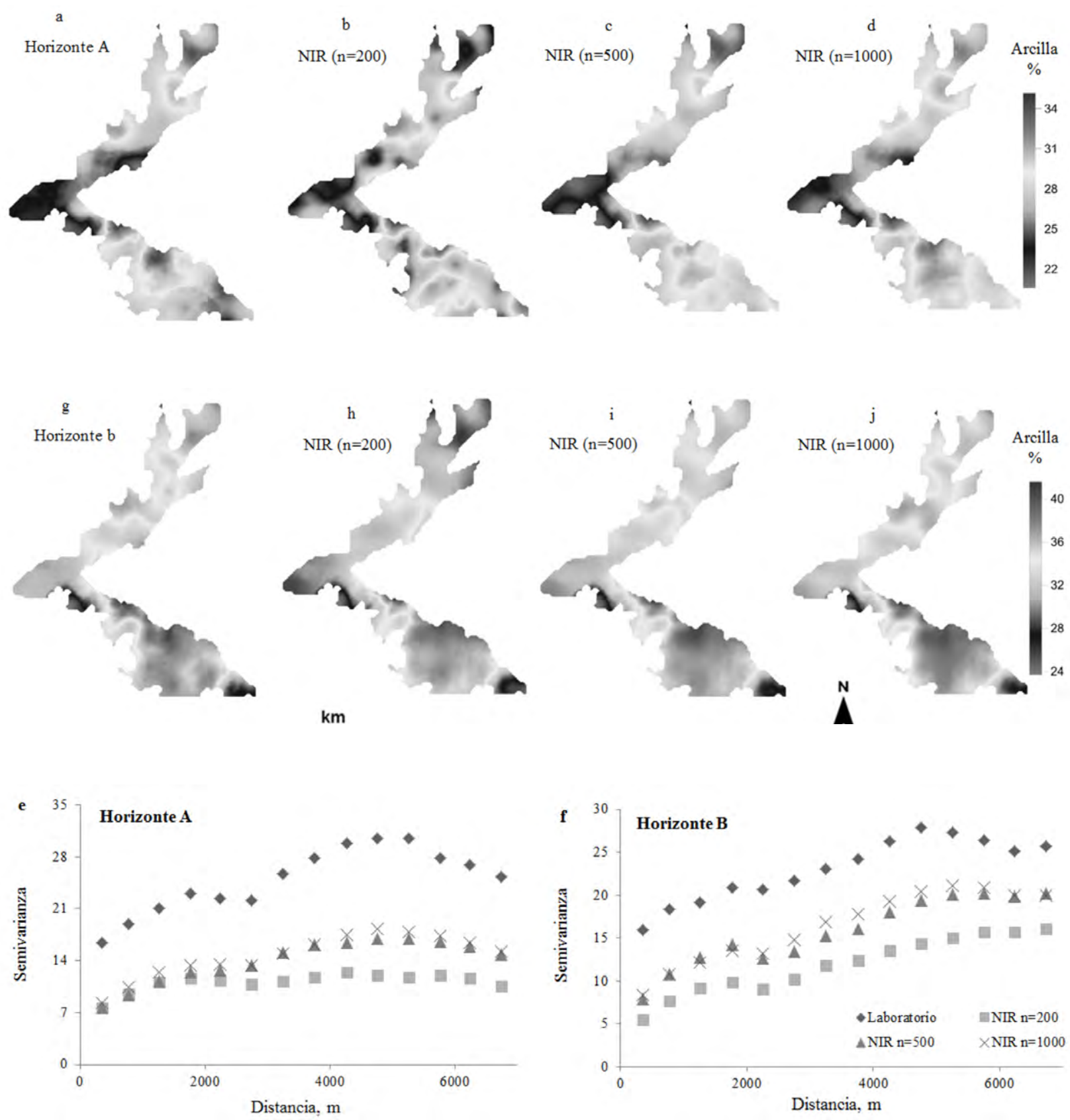

Figura 5. Mapas digitales obtenidos para el contenido de arcilla en los horizontes A (superior) y B (inferior), a y g a partir de los datos de laboratorio y b, c, d, h, i, j a partir de los modelos espectrales obtenidos con diferentes números de muestras de calibración; e y f corresponden a los semivariogramas para la obtención de cada mapa digital para los dos horizontes. 
Tabla 3. Parámetros de los semivariogramas teóricos obtenidos para Arcilla a partir de los datos de laboratorio (medidos) y estimados a partir del modelo (NIR).

\begin{tabular}{lllllllc}
\hline \multicolumn{1}{c}{ Datos } & Modelo & $\mathbf{C}_{\mathbf{0}}$ & $\mathbf{C}_{\mathbf{0}}+\mathbf{C}_{\mathbf{1}}$ & $\mathbf{a}(\mathbf{m})$ & $\mathbf{R}^{\mathbf{2}}$ & $\mathbf{C V C}$ & $\mathbf{C} /\left(\mathbf{C}_{\mathbf{0}}+\mathbf{C}_{\mathbf{1}}\right)$ \\
\hline & & \multicolumn{7}{c}{ Horizonte $\mathbf{A}$} \\
\hline Medidos & Esférico & 14.32 & 28.68 & 5000 & 0.85 & 0.98 & 0.50 \\
NIR $_{200}$ & Esférico & 5.79 & 11.59 & 1660 & 0.85 & 0.94 & 0.50 \\
NIR $_{500}$ & Esférico & 6.97 & 16.27 & 4700 & 0.96 & 0.99 & 0.57 \\
NIR $_{1000}$ & Esférico & 8.22 & 17.01 & 5060 & 0.90 & 1.03 & 0.52 \\
\hline \multicolumn{7}{c}{ Horizonte B } \\
\hline Medidos & Esférico & 13.5 & 27.01 & 5520 & 0.94 & 1.01 & 0.50 \\
NIR $_{200}$ & Esférico & 5.68 & 16.76 & 8350 & 0.97 & 1.05 & 0.66 \\
NIR $_{500}$ & Esférico & 8.25 & 20.65 & 7410 & 0.94 & 1.05 & 0.60 \\
NIR $_{1000}$ & Esférico & 7.64 & 20.54 & 6100 & 0.97 & 1.05 & 0.63 \\
\hline
\end{tabular}

$\mathrm{C}_{0}$ : efecto pepita, $\mathrm{C}_{0}+\mathrm{C}_{1}$ : meseta, a: rango o alcance, $\mathrm{R}^{2}$ : coeficiente de determinación.

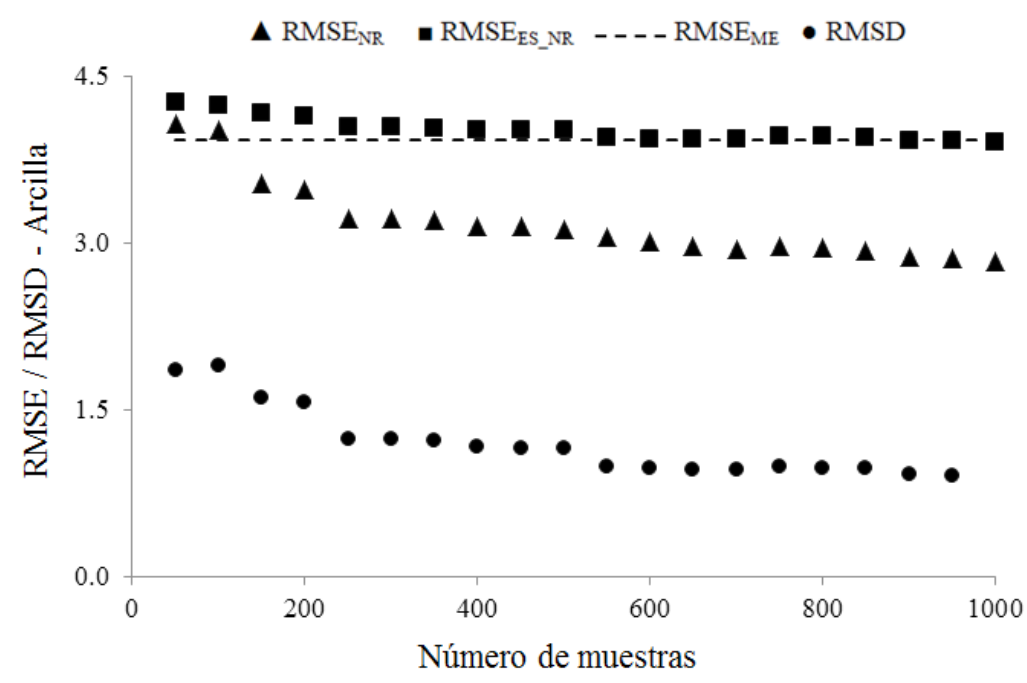

Figura 6. Variación de los parámetros $\mathrm{RMSE}_{\mathrm{NIR}^{\prime}} \mathrm{RMSE}_{\mathrm{ES}_{-} \mathrm{NIR}} \mathrm{RMSE}_{\mathrm{ME}}$ y $\mathrm{RMSD}$ según el número de muestras usado en la calibración de los modelos.

Para los mapas digitales, se consideró la variación del RMSE ${ }_{\mathrm{NIR}}$ RMSE $_{\mathrm{ES}_{-} N R^{\prime}}$ RMSE $\mathrm{ME}_{\mathrm{ME}}$ y RMSD en función del número de muestras para determinar la propagación del error.

\section{DISCUSIÓN}

El limo, fracción que prevalece en el suelo, presentó baja variabilidad para los dos horizontes. En general, las fracciones del suelo presentan baja o media variabilidad, donde la menor variabilidad se manifiesta en la partícula que predomina (Rodríguez-Vásquez et al., 2008; Peña et al., 2009) (tabla 1). Resultados similares de textura fueron reportados para oxisoles por Orjuela-Matta et al., (2010), donde la textura varía de franca a arcillosa, debido a la intensa meteorización física (tabla 1), donde el arrastre superficial de las partículas finas del suelo favorece la prevalencia del limo. 
Para los contenidos de arena y limo se obtuvieron modelos con baja representatividad $\left(R^{2}\right.$ de 0.41 y 0.34 , respectivamente, tabla 2). Curcio et al., (2013) reportan valores mayores, para arena $\mathrm{R}^{2}$ de 0.80 y para limo $\mathrm{R}^{2}$ de 0.60 . Igualmente Casa et al., (2013) reportan valores $R^{2}$ para limo y arcilla de 0.63 y 0.79 respectivamente. En cuanto al RMSE se registraron valores para arena de $4.75 \%$ y limo de $4.04 \%$, menores a los reportados por Curcio et al., (2013) de $7.7 \%$ para arena y $7.2 \%$ para limo.

Los valores de RPD fueron inferiores a 1.5 tanto para la calibración y validación del modelo (tabla 2), por lo que el uso de estos modelos no es apropiado. Sin embargo, Casa et al., (2013) obtiene modelos con buena capacidad de predicción con valores de RPD de 1.6 para limo y 2.1 para arena. Para estos dos contenidos se observó que las validaciones cruzadas son dispersas, con presencia de valores atípicos y baja correlación entre los datos observados y los valores predichos por el modelo (figura 2 a y b), lo que corrobora el bajo poder de predicción de los modelos obtenidos para estos dos contenidos de partículas. En los grupos de validación se obtienen valores menores de $\mathrm{R}^{2}$ en comparación con los grupos de calibración, en los ajustes para la validación cruzada.

Es posible que algunas de las diferencias mencionadas anteriormente entre los modelos obtenidos y los reportados por otros autores, así como los comportamientos observados en las validaciones cruzadas para los contenidos de arena y limo, sean consecuencia de una superposición de algún efecto diferente que perturba las características de absorción en algunas regiones del espectro. Además, la presencia de minerales como el cuarzo resultan insensibles a la espectroscopía VIS-NIR, pues sus características de absorción pueden ser observadas como vibraciones intensas, fundamentales en la gama del infrarrojo medio (Viscarra-Rossel et al, 2009). Para los suelos de Carimagua la fracción arena tiene un dominio de cuarzo en todos los horizontes.

Cabe mencionar que algunos de los estudios señalados utilizaron diferentes tipos de suelo, algoritmos de muestreo y diferentes tratamientos de las respuestas espectrales (Camargo et al, 2015). De igual modo, los bajos contenidos de los atributos analizados inciden también en un mayor error analítico a nivel de laboratorio. Según Mouazen et al., (2010) factores ambientales, como la luz, la humedad relativa y la temperatura también inciden en la obtención de las respuestas espectrales, afectando regiones del espectro que están estrechamente relacionadas con algunas propiedades en particular. Es importante considerar que el uso de diferentes técnicas estadísticas (empleadas de manera individual o grupal) pueden mejorar el desempeño predictivo de los modelos propuestos. Así mismo, explorar otras regiones espectrales, como el visible o la región del infrarrojo medio-MIR puede permitir la elaboración de mejores modelos para varias propiedades del suelo (Viscarra Rossel et al., 2006).

El modelo para el contenido de arcilla mostró un $\mathrm{R}^{2}$ alto (0.76), indicando un buen desempeño predictivo cuando se utilizan 1000 muestras para la calibración del modelo. El coeficiente de determinación resulta aceptable y concuerda con los valores encontrados por Curcio et al. (2013), Casa et al. (2013), Terra et al. (2015) y Camargo et al. (2015) que oscila entre 0.58 y 0.87 para el contenido de arcilla. Es importante resaltar que en algunos de los estudios mencionados se trabajó con la región VIS-NIR del espectro y tipos de suelo distintos. También se trató con muestras de suelo que presentan contenidos de humedad diferentes, por lo tanto la presencia de agua en el suelo puede ayudar a las predicciones de contenido de arcilla, ya que son propiedades que están relacionadas (por ejemplo, hay presencia de agua y minerales propios de la arcilla entre los 1800 y 2000 nm), así como algunas de las características de absorción (por ejemplo, los relacionados con óxidos de hierro) en el rango del infrarrojo visible (Viscarra-Rossel et al., 2009).

Al aumentar el número de muestras usadas para calibrar los modelos se evidencia que los valores de $\mathrm{R}^{2}$ y RPD aumentan mientras que el valor de RMSE disminuye. Es decir, a mayor número de muestras se obtienen modelos más robustos (figura 3). 
Buscando el menor número de muestras que represente el $95 \%$ de la meseta, para $\mathrm{R}^{2}$ se obtuvo que el número de muestras para calibrar el modelo fue de 455 y para RMSE fue de 517. En el caso del RPD se buscaron dos criterios en los cuales se presentan valores aceptables de predicción, dando como resultado que para obtener un RPD $>1.5$ se requiere de 50 muestras y para un $\mathrm{RPD}>2.0$ se requiere de 588 muestras para la calibración de los modelos. Por lo tanto, se obtuvo que para el contenido de arcilla el número de muestras con el que es posible obtener un modelo óptimo con una capacidad de predicción aceptable es de 517 muestras para calibración. Sin embargo, para esta propiedad Ramírez-López et al. (2014) reportan que al menos con 100 muestras es posible la calibración óptima de modelos, utilizando métodos diferentes para la determinación de este atributo.

La validación cruzada entre los datos medidos y los estimados para el contenido de arcilla evidencia el buen ajuste que presenta esta variable (figura 2c), se muestra una menor dispersión con valores altos de $\mathrm{R}^{2}$, tanto para la calibración como para la validación de los modelos, donde se verifica que los grupos de calibración y validación presentan comportamientos similares.

El mayor pico presente en la arcilla se observa a $1900 \mathrm{~nm}$, seguido de picos presentes de los $2200 \mathrm{~nm}$ a los $2400 \mathrm{~nm}$ (figura 4). Franceschini et al. (2013) señalan que para arcilla y arena se presentan picos en la región de 1200 nm, 1900 $\mathrm{nm}$ y $2200 \mathrm{~nm}$ debido a la presencia de filosilicatos (como caolinita) y gibsita para oxisoles y ultisoles en la región de Mato Grosso, Brasil. Para esta investigación, en esta región del espectro se manifiesta la caolinita. Según Genú \& Demattê (2012), la caolinita se manifiesta en los picos ubicados a 1900 y 2200 nm para diferentes clases de suelo. Para los suelos de Carimagua, este es el mineral predominante en la fracción arcilla.

Como se encontró que el modelo de arcilla obtuvo buen comportamiento predictivo, se realizó el análisis geoestadístico para este tipo de partícula. Para los semivariogramas del contenido de arcilla se obtuvieron modelos teóricos apropiados con $\mathrm{R}^{2}$ mayores de 0.85 y CVC próximos a 1 (tabla 3). Para los datos estimados de los modelos NIR a partir de diferente número de muestras, la estructura espacial de los datos se mantiene. Se evidencia que con un bajo número de muestras en los modelos de calibración el alcance varía significativamente del resto. En general, este comportamiento es reportado por Cobo et al. (2010) y Ramírez-López et al. (2014), donde se exponen las ventajas del uso de los modelos espectrales en la elaboración de mapas digitales.

Para los horizontes $\mathrm{A}$ y $\mathrm{B}$ se observaron menores valores de pepita $\left(C_{0}\right)$ y meseta $\left(C_{0}+C_{1}\right)$ para los semivariogramas obtenidos con los modelos NIR (tabla 3). Por lo tanto, se pueda afirmar que en los datos estimados la varianza es menor. Los resultados para el contenido de arcilla indican que a partir de los datos estimados de los modelos NIR el error de medición puede ser menor, dado que el valor de $\mathrm{C}_{0}$ se relaciona directamente con el error propio del proceso de muestreo, así como con la medición de la propiedad de interés.

Para el contenido de arcilla se obtuvieron alcances de $1600 \mathrm{~m}$ a $8600 \mathrm{~m}$, estos resultados indican que no es posible disminuir la densidad de muestreo al menos para el horizonte $B$ que presenta los alcances mayores (tabla 3). Sin embargo, para el horizonte A sí es posible y las distancias entre puntos pueden variar de $1000 \mathrm{~m}$ a $5000 \mathrm{~m}$ para identificar los patrones de variabilidad. Los datos estimados a partir del modelo con 500 muestras presentan valores similares de alcance al obtenido con los datos medidos para ambos horizontes. Por lo tanto, se verifica la relación entre el número de muestras usadas para calibrar el modelo y la distancia en la que es posible que una propiedad tenga relación espacial.

De acuerdo al grado de dependencia espacial (GDE), definido por la relación $\mathrm{C}_{1} /\left(\mathrm{C}_{0}+\mathrm{C}_{1}\right)$, el contenido de arcilla presentó un grado de dependencia espacial moderado tanto para los semivariogramas obtenidos a partir de los datos medidos como de los estimados a partir de los modelos. Cabe resaltar que para el horizonte B, el GDE para 
datos estimados es mayor que para los datos medidos. Se verifica que el GDE resultante de los semivariogramas obtenidos a partir de los modelos, es consistente con el obtenido a partir de los datos medidos (tabla 3).

Al comparar los mapas digitales (elaborados a partir de datos medidos) y los mapas espectro digitales, se observó un comportamiento similar al de los semivariogramas, pues hay influencia del número de muestras de calibración. Cobo et al. (2010) y Ramírez-López et al. (2014) reportan comportamientos similares, donde un bajo número afecta el análisis espacial, ya que la densidad de muestreo cambia y la correspondencia entre mapas también. En la figura 5 se evidencia que los mapas elaborados con los grupos de 200 y 500 muestras presentan mayor semejanza al mapa de datos medidos para el contenido de arcilla en ambos horizontes. Cantarella et al. (2006) afirman que la diferencia entre mapas para la determinación de una misma propiedad puede estar asociada a errores de muestreo, que son generalmente mayores que los errores derivados del análisis de suelos.

Es claro que la distancia de muestreo depende de la propiedad que se analice. Sin embargo, Demattê et al. (2006) exponen más criterios a considerar. Estos autores verificaron para un oxisol de São Paulo (Brasil), que la densidad de muestreo mínima requerida en la práctica es de una muestra por hectárea, lo cual inviabiliza la elaboración del mapa debido al alto costo de los análisis de suelos. Para que sea económicamente viable la densidad de muestreo debe ser de una muestra por cada 4 ha. Debido a la alta correspondencia entre mapas digitales y mapas espectro digitales, es posible decir que técnicas como la espectroscopía de reflectancia difusa pueden minimizar limitantes de este tipo y ser de gran utilidad en campos como la agricultura de precisión, planes de ordenamiento territorial, levantamiento de suelos, unidades geomorfológicas e identificación de unidades de manejo agronómico.

Es evidente que los parámetros considerados para precisar la propagación del error resultan ser mayores cuando se emplea un bajo número de muestras para la calibración del modelo de contenido de arcilla y que decrecen a medida que el número de muestras aumenta, afectando de manera directa la correspondencia entre mapas interpolados a partir de kriging ordinario (figura 6).

Se observó que el RMSE $\mathrm{ES}_{\text {_NIR }}$ presenta un comportamiento similar al RMSE ${ }_{\mathrm{ME}}$. Además, los errores entre los valores medidos comparados con los valores obtenidos por kriging a partir de los resultados de los modelos espectrales son bajos (RMSD $<$ RMSE). Por esto, es posible afirmar que a partir de un número de muestras específico se pueden obtener mapas digitales y espectro digitales altamente correlacionados con bajos errores (figura 6). Según Kuang \& Mouazen (2013), es recomendable verificar este comportamiento para definir la calidad de los mapas espectro-digitales.

Sin embargo, es necesario tener en cuenta otros aspectos, como la finalidad del estudio de suelos para definir el número mínimo de muestras en la calibración de modelos del contenido de arcilla y en general de la propiedad de interés, pues es posible determinar este número mínimo en base a los parámetros de los modelos de calibración. Aunque, si también se busca la elaboración de mapas espectro digitales mediante kriging, se hace necesario considerar las distancias de muestreo adecuadas obtenidas a partir de los semivariogramas y analizar los resultados de los errores y la propagación de estos (Cobo et al., 2010).

\section{CONCLUSIONES}

La técnica de espectroscopía NIR resulta útil para determinar el contenido de arcilla de un oxisol, los resultados encontrados indican que los análisis de laboratorio se pueden sustituir en gran parte por los modelos espectrales. Los resultados mostraron que los datos obtenidos a partir de los modelos NIR pueden integrarse directamente en evaluaciones geoestadísticas para el contenido de arcilla en un oxisol. 
En el caso de los contenidos de arena y limo no se obtuvieron modelos aceptables. Por lo tanto, sería conveniente mejorar el modelo para que en el futuro los análisis de laboratorio puedan ser remplazados para esta clase de suelo. Se recomienda el estudio de textura para un oxisol en otras regiones del espectro como VIS y MIR, elaboración de modelos a diferentes contenidos de humedad del suelo, así como el uso de diferentes técnicas estadísticas para el tratamiento de las curvas espectrales.

\section{REFERENCIAS BIBLIOGRÁFICAS}

Bellinaso, H., Demattê, J.A.M. \& Romeiro, S.A. (2010). Soil spectral library and its use in soil. Revista Brasileira de Ciência do Solo, 34, 861-870.

Bisquerra, R. (2004). Metodología de la investigación educativa. Madrid: La Muralla.

Camacho-Tamayo, J. H., Rubiano S., Y., \& Hurtado S., M. (2014). Near-infrared (NIR) diffuse reflectance spectroscopy for the prediction of carbon and nitrogen in an Oxisol. Agronomía Colombiana, 32(1), 86-94.

Camargo, L. A., Marques Júnior, J., Barrón, Vidal, Ferracciú, L. R., Barbosa, R. S., \& Pereira, G. T. (2015). Mapping of clay, iron oxide and absorbed phosphate in Oxisols using diffuse reflectance spectroscopy. Geoderma, 251-252, 124-132.

Cambardella, C.A., Moorman, T.B., Novak, J.M., Parkin, T.B., Karlen, D.L., Turco, R.F., \& Konopka, A.E. (1994). Field-scale variability of soil properties in Central lowa Soils. Soil Science Society of American Journal, 5, 1501-1511.

Cantarella, H., Quaggio, J. A., Raij, B. Van., \& Abreu, M.F. (2006). Variability of soil analysis in commercial laboratories: implications for lime and fertilizer recommendations. Communications in Soil Science and Plant Analysis, 37, 2213-2225.

Carvalho Junior, W. de, Chagas, C. Da S., Muselli, A., Pinheiro, H. S. K., Pereira, N. R., \& Bhering, S. B. (2014). Método do hipercubo latino condicionado para a amostragem de solos na presença de covariáveis ambientais visando o mapeamento digital de solos. Revista Brasileira de Ciência do Solo, 38(2), 386-396.

Casa, R., Castaldi, F., Pascucci, S., Palombo, A., \& Pignatti, S. (2013). A comparison of sensor resolution a calibration strategy for soil texture estimation from hyperespectral remote sensing. Geoderma, 197-198, 17-26.

Cobo, J.G., Dercon, G., Yekeye, T., Chapungu, L., Kadzere, C., Murwira, A., Delve, R., \& Cadisch, G. (2010). Integration of mid-infrared spectroscopy and geostatistics in the assessment of soil spatial variability at landscape level. Geoderma, 158, 398-411.

Curcio, D., Ciraolo, G., D' Asaro, F., \& Minacapilli, M. (2013). Prediction of soil texture distributions using VNIR-SWIR reflectance spectroscopy. Procedia Environmental Sciences, 19, 494-503.

Demattê, J.A.M., Morelli, J., \& Demattê, J.L.I. (2006). Nem tanta precisão. Idea News, 70, 52-60.

Diggle, P., \& Ribeiro, J. (2000). Model Based Geostatistics. 1ed. São Paulo: Associação Brasileira de Estatística. 129 p.

Franceschini, M. H. D., Demattê, J. A. M., Sato, M. V., Vicente, L. E., \& Grego, C. R. (2013). Abordagens semiquantitativa e quantitativa na avaliação da textura do solo por espectroscopia de reflectância bidirecional no VIS-NIR-SWIR. Pesquisa Agropecuária Brasileira, 48(12), 1569-1582.

García Durán, Obed. (2009). Carimagua: La investigación y el desarrollo en ecosistemas de baja fertilidad. Revista Colombiana de Ciencias Pecuarias, 22(1), 74-78.

Genú, A.M., \& Demattê, J.A.M. (2012). Espectrorradiometria de solos e comparação com sensores orbitais. Bragantia, 71, 82-89.

Goovaerts, P. (1998). Geostatistical tools for characterizing the spatial variability of microbiological and physico-chemical soil properties. Biology and Fertility of Soils, 27(4), 315-334.

Johann, J.A., Silva, M.C.A., Uribe-Opazo, M.A., \& Dalposso, G.H. (2010). Variabilidade Espacial da rentabilidade, perdas na colheita e produtividade do Feijoeiro. Engenharia Agrícola, 30, 700-714. 
Kuang, B., \& Mouazen, A.M. (2013). Effect of spiking strategy and ratio on calibration of on-line visible near infrared soil sensor for measurement in European farms. Soil and Tillage Research, 128,125-136.

Lacasta, C., Meco, R., \& Maire, N. (2005). Evolución de las producciones y de los parámetros químicos y bioquímicos del suelo, en un agrosistema de cereales sometido a diferentes manejos de suelo durante 21 años. El reto de la Agricultura y Medio Ambiente, la Energía y la Nueva Política Agraria Común. Congreso Internacional sobre Agricultura de Conservación, 429-436.

Ministerio de Agricultura. (2016). Evaluaciones Agropecuarias del Ministerio de Agricultura y DesarroIlo Rural. Recuperado de: http://www.agronet.gov. co/estadistica/Paginas/default.aspx

Mouazen, A.M., Kuang, B., De Baerdemaeker, J., \& Ramon, H. (2010). Comparison among principal component, partial least squares and back propagation neural network analyses for accuracy of measurement of selected soil properties with visible and near infrared spectroscopy. Geoderma, 158, 23-31.

Nanni, M.R., \& Demattê, J.A.M. (2006). Spectral reflectance methodology in comparison to tradicional soil analysis. Soil Science Society of America Journal, 70, 393-407.

Orjuela-Matta, H. M., Rubiano Sanabria, Y., \& Camacho-Tamayo, J. H. (2010). Comportamiento de la Infiltración en un Oxisol. Revista U.D.C.A Actualidad \& Divulgación Científica, 13(2), 31-39.

Peña, R., Rubiano, Y., Peña, A., \& Chaves, B. (2009). Variabilidad especial de los atributos de la capa arable de un Inceptisol del piedemonte de la Cordillera Oriental (Casanare, Colombia). Agronomía Colombiana, 27, 111-120.

Ramírez, E., Anaya, A. M., \& Mariscal, G. (2005). Predicción de la composición química del grano de sorgo mediante espectroscopía de reflectancia en el infrarrojo cercano (NIRS). Revista Mexicana de Ciencias Pecuarias, 43, 1-11.

Ramírez-López, L., Schmidt, K., Behrens, T., Wesemael, B., Demattê, J.A.M., \& Scholten T. (2014).
Sampling optimal calibration sets in soil infrared. Geoderma, 226-227, 140-150.

Rodríguez-Vásquez, A.F., Aristizabal-Castillo, A.M., \& Camacho-Tamayo, J.H. (2008). Variabilidad espacial de los modelos de infiltración de Philip y Kostiakov en un suelo ándico. Engenharia. Agrícola, 28, 64-75.

Sousa Junior, J.G., Demattê, J.A.M., \& Araújo, S.R. (2011). Modelos espectrais terrestres e orbitais na determinação de teores de atributos dos solos: potencial e custos. Bragantia, 70, 610-621.

Stenberg, B., Viscarra Rossel, R.A., Mouazen, A.M., \& Wetterlindd, J. (2010). Visible and near infrared spectroscopy in soil science. Advances in Agronomy, 107, 163-215.

Terra, F. S., Demattê, J. A., \& Viscarra Rossel, R. A. (2015). Spectral libraries for quantitative analyses of tropical Brazilian soils: Comparing VIS -NIR and mid- IR reflectance data. Geoderma, 255-256, 81-93.

Viscarra Rossel, R., Walvoort, D., McBratney, A., Janik, L., \& Skjemstad, J. (2006). Visible, near infrared, mid infrared or combined diffuse reflectance spectroscopy for simultaneous assessment of various soil properties. Geoderma, 131, 59-75.

Viscarra Rossel, R.A. (2008). ParLeS: Software for chemometric analysis of spectroscopic data. Chemometrics and Intelligent Laboratory Systems, 90, 72-83.

Viscarra Rossel, R.A., Catttle, S.R., Orteaga, A., \& Fouad, Y. (2009). In situ measurements of soil solour, mineral composition and clay content by VIS - NIR spectroscopy. Geoderma, 150, 253-266.

Webster, R., \& Oliver, M.A. (2007). Geostatistics for environmental scientists. Hoboken, NJ: Editorial John Wiley \& Sons Inc. 109p.

White, R.E. (2005). Principles and practice of soil science: the soil as a natural resource. Cuarta edición. Oxford: Blackwell. 384 p.

Wold, S., Sjöström, M., \& Eriksson, L. (2001). PLS-regression: la basic tool of chemometrics. Chemometrics and Intelligent Laboratory Systems, 58,109-130.

\section{(c) $(1) \Theta$}

